\title{
PERTURBATIONS OF LIMIT-CIRCLE EXPRESSIONS
}

\author{
THOMAS T. READ
}

\begin{abstract}
It is shown that for any limit-circle expression $L(y)$ $=\sum_{j=0}^{n} p_{j} y^{(j)}$, any sequence of disjoint intervals $\left\{\left[a_{k}, b_{k}\right]\right\}_{k=1}^{\infty}$ such that $a_{k} \rightarrow \infty$ as $k \rightarrow \infty$, and any $i \leqslant n-1$, there is an expression $M(y)$ $=\sum_{j=0}^{n} q_{j} y^{(j)}$ such that $q_{i}=p_{i}$ except on $\cup\left(a_{k}, b_{k}\right), q_{j}=p_{j}$ for all $j \neq i$, and such that $M$ is not limit-circle.
\end{abstract}

An $n$th order ordinary differential expression $L(y)=\sum_{j=0}^{n} p_{j} y^{(j)}$, where each $p_{j}$ is a complex-valued function on $[0, \infty)$ with continuous $j$ th derivative and $p_{n}$ is zero-free, is said to be limit-circle if all solutions of $L(y)=0$ and all solutions of $L^{+}(y)=0$ lie in $L^{2}(0, \infty)$. Here $L^{+}$is the formal (Lagrange) adjoint of $L$. The smoothness assumptions on the $p_{j}$ 's ensure the existence of $L^{+}$as a differential expression. They can be avoided by suitable use of quasidifferential expressions. See [4].

We shall show that the limit-circle property depends on the behavior of the coefficient functions on the entire interval. More precisely, we have

THEOREM. Let $L(y)=\sum_{j=0}^{n} p_{j} y^{(j)}$ be limit-circle, let $\left\{\left[a_{k}, b_{k}\right]\right\}_{k=1}^{\infty}$ be any sequence of pairwise disjoint intervals such that $a_{k} \rightarrow \infty$ as $k \rightarrow \infty$, and let $i \leqslant n-1$. Then there is an expression $M(y)=\sum_{j=0}^{n} q_{j} y^{(j)}$ such that $q_{i}=p_{i}$ except on $\cup_{k=1}^{\infty}\left(a_{k}, b_{k}\right), q_{j}=p_{j}$ for each $j \neq i$, and such that $M$ is not limitcircle.

REMARK. If $L$ is a second order real formally symmetric expression, then $L$ is either limit-circle or limit-point. Thus the theorem asserts in this case that for any limit-circle expression $-\left(r y^{\prime}\right)^{\prime}+p y$ there is a limit-point expression $-\left(r y^{\prime}\right)^{\prime}+q y$ such that $q=p$ on the complement of a prescribed sequence of intervals. This extends, in part, a result of Eastham and Thompson [1] who show that for a certain class of limit-circle expressions the above conclusion holds with the added property that $q$ is monotonic. Our assertion, for second order real formally symmetric expressions, can also be deduced from a limitpoint criterion of Ismagilov [2] (for leading coefficient 1) and Knowles [3].

Proof. The proof is based on the observation that a necessary (though far from sufficient) condition for $L$ to be limit-circle is that there exist a positive constant $K$ such that

$$
\|L(f)\| \geqslant K\|f\|
$$

Presented to the Society, January 23, 1976; received by the editors February 7, 1975.

AMS (MOS) subject classifications (1970). Primary 34B20.

Key words and phrases. nth order ordinary differential expression, Weyl theory, limit-circle condition.

○ American Mathematical Society 1976 
for all $C^{\infty}$ functions $f$ with compact support in the interior of $[0, \infty)$. Here $\|\cdot\|$ denotes the usual norm in $L^{2}(0, \infty)$. This may be seen as follows. There are solutions $\varphi_{1}, \ldots, \varphi_{n}$ of $L(y)=0$ and $\psi_{1}, \ldots, \psi_{n}$ of $L^{+}(y)=0$ such that $V(x, t)=\sum_{j=1}^{n} \varphi_{j}(x) \psi_{j}(t)$ has the property that for any $g \in L^{2}(0, \infty), f(x)$ $=\int_{0}^{x} V(x, t) g(t) d t \quad$ satisfies $L(f)=g$ and $f(0)=f^{\prime}(0)=\cdots=f^{(n-1)}(0)$ $=0$. Thus a restriction of this integral operator is the inverse of the operator determined by the differential expression $L$ on the linear space of $C^{\infty}$ functions with compact support in the interior of $[0, \infty)$. From the assumption that $L$ is limit-circle it is clear that this integral operator is a Hilbert-Schmidt operator and so, in particular, continuous. Thus the inequality (1) is valid for some positive $K$.

Now suppose that $\left\{\left[a_{k}, b_{k}\right]\right\}_{k=1}^{\infty}$ is given. We complete the proof first for $i=0$. From the above observation it suffices to define $M$ on $\bigcup_{k=1}^{\infty}\left[a_{k}, b_{k}\right]$ so that for each $k$ there is a $C^{\infty}$ function $f_{k}$ supported on the interior of $\left[a_{k}, b_{k}\right]$ such that $\left\|M\left(f_{k}\right)\right\|<(1 / k)\left\|f_{k}\right\|$. It will be convenient to adopt the notation $L_{1}(y)=\sum_{j=1}^{n} p_{j} y^{(j)}=L(y)-p_{0} y$. We may consider the intervals independently and so must only show that given any interval $[a, b]$ and any $\varepsilon>0$ there is a function $q_{0}$ on $[a, b]$ such that $q_{0}(a)$ and $q_{0}(b)$ have prescribed values (to make $q_{0}$ continuous on $[0, \infty)$ ), and a $C^{\infty}$ function $f$ supported on the interior of $[a, b]$ such that $M(y)=L_{1}(y)+q_{0} y$ satisfies $\|M(f)\|<\varepsilon\|f\|$.

Let $f$ be any fixed $C^{\infty}$ function supported on $[\alpha, \beta] \subset(a, b)$ such that $f(x)>0$ for $\alpha<x<\beta$ and $\|f\|=1$. Choose $\gamma$ and $\delta$ with $\alpha<\gamma<\delta<\beta$ so that

$$
\int_{\alpha}^{\gamma}\left|L_{1}(f)\right|^{2} d t+\int_{\delta}^{\beta}\left|L_{1}(f)\right|^{2} d t<\varepsilon^{2} / 4 .
$$

Define $q_{0}$ on $[\gamma, \delta]$ by $q_{0}=-L_{1}(f)(x) / f(x)$. If $q_{0}$ is then extended first to $[\alpha, \beta]$ so that it vanishes outside a sufficiently small neighborhood of $[\gamma, \delta]$, and then to $[a, b]$ so that $q_{0}(a)$ and $q_{0}(b)$ have the prescribed values, then

$$
\int_{\alpha}^{\gamma}\left|q_{0} f\right|^{2} d t+\int_{\delta}^{\beta}\left|q_{0} f\right|^{2} d t<\varepsilon^{2} / 4
$$

Thus

$$
\|M(f)\|^{2}=\int_{\alpha}^{\gamma}|M(f)|^{2} d t+\int_{\delta}^{\beta}|M(f)|^{2} d t<\varepsilon^{2},
$$

and the proof is complete when $i=0$.

For $i>0$ the above construction may be repeated with the obvious modifications. The only additional complication is that $f^{(i)}$ will have zeros in the interior of its support. However by proper choice of $f$ we may assume that there are only finitely many of these, so that we may define $q_{i}(x)$ outside the union of small neighborhoods of these points so that $M(f)=0$, and then extend $q_{i}$ to $[a, b]$ as before.

\section{REFERENCES}

1. M. S. P. Eastham and M. L. Thompson, On the limit-point limit-circle classification of second order ordinary differential equations, Quart. J. Math. Oxford Ser. (2) 24 (1973), 531-535.

2. R. S. Ismagilov, On the self-adjointness of the Sturm-Liouville operator, Uspehi Mat. Nauk 18 (1963), no. 5 (113), 161-166. (Russian) MR 27 \#4979. 
3. I. Knowles, Note on a limit-point criterion, Proc. Amer. Math. Soc. 41 (1973), 117-119. MR 47 \#8964.

4. A. Zettl, Formally self-adjoint quasi-differential operators, Rocky Mountain J. Math. 5 (1975), 453-474.

Department of Mathematics, University of Dundee, Dundee, Scotland

Current address: Department of Mathematics and Computer Science, Western Washington State College, Bellingham, Washington 98225 